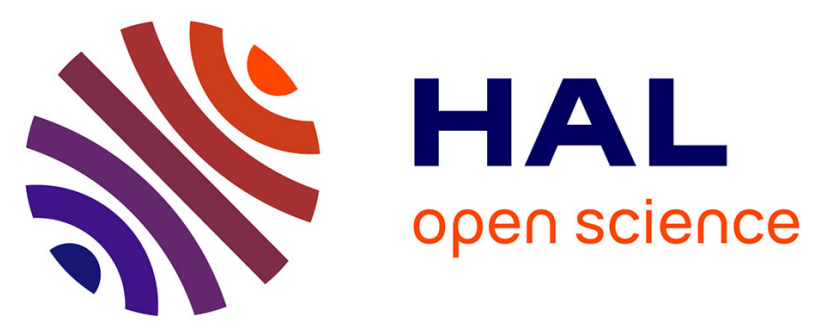

\title{
A Simultaneous, Multidisciplinary Development and Design Journey - Reflections on Prototyping
}

Achim Gerstenberg, Heikki Sjöman, Thov Reime, Pekka Abrahamsson, Martin Steinert

\section{- To cite this version:}

Achim Gerstenberg, Heikki Sjöman, Thov Reime, Pekka Abrahamsson, Martin Steinert. A Simultaneous, Multidisciplinary Development and Design Journey - Reflections on Prototyping. 14th International Conference on Entertainment Computing (ICEC), Sep 2015, Trondheim, Norway. pp.409-416, 10.1007/978-3-319-24589-8_33 . hal-01758417

\section{HAL Id: hal-01758417 \\ https://hal.inria.fr/hal-01758417}

Submitted on 4 Apr 2018

HAL is a multi-disciplinary open access archive for the deposit and dissemination of scientific research documents, whether they are published or not. The documents may come from teaching and research institutions in France or abroad, or from public or private research centers.
L'archive ouverte pluridisciplinaire HAL, est destinée au dépôt et à la diffusion de documents scientifiques de niveau recherche, publiés ou non, émanant des établissements d'enseignement et de recherche français ou étrangers, des laboratoires publics ou privés. 


\title{
A Simultaneous, Multidisciplinary Development and Design Journey - Reflections on Prototyping
}

\author{
Achim Gerstenberg ${ }^{\mathrm{a}}$, Heikki Sjöman ${ }^{\mathrm{a}}$, Thov Reime ${ }^{\mathrm{a}}$, Pekka Abrahamsson ${ }^{\mathrm{b}}$, Martin \\ Steinert ${ }^{\mathrm{a}}$ \\ ${ }^{a}$ Dep. of Eng. Design and Mat., ${ }^{b}$ Dep. of Comp. and Info. Sc. \\ NTNU, Høgskoleringen 1, 7491 Trondheim, Norway \\ \{Achim.Gerstenberg, Heikki.Sjoman,Thovr, Pekkaa, Martin.Steinert, \}@ntnu.no,
}

\begin{abstract}
This paper proposes a wayfaring approach for the early concept creation stage of development projects that have a very high degree of intended innovation and thus uncertainty. The method is supported by a concrete game design example involving the development of a tangible programming interface for virtual car racing games. We focus onto projects that not only have high degrees of freedom, for example in terms of reframing the problem or iterating the final project vision, but are also complex in nature. For example, these can be projects that allow for the exploration and exploitation of unknown unknowns and serendipity findings. Process wise we are primarily focusing onto the early stage that precedes the requirement fixation, which we see as more dynamic and evolutionary in nature. The core conceptual elements that we have derived from the development experiences are: simultaneous prototyping in multiple disciplines (such as computer science, electronics and mechanics and engineering in general, abductive learning based on the outcome of rapid cycles of designing, building and testing prototypes (probing), and the importance of including all the involved disciplines (knowledge domains) from the beginning of the project on.
\end{abstract}

\section{Introduction}

To innovate incrementally is hard, to innovate "radically" harder still. Many an engineering project is fixating their requirements very early and then focus onto executing these predefined (and often unproven) specs as fast, as good, and as cost effective as possible. The usual outcome is a cost and/or time overrun if the innovative specs are to be met or a decrease in result quality. In a sense people perceive the innovation game often as a game under certainty with fixed variables and attribute values, fixed rules and thus predictable outcomes, hence it can be modeled, simulated and optimized. We argue that the innovation game is a game under uncertainty, with unknown unknowns that need to be discovered, evaluated and then discarded or embodied. The game is also played in a dynamic environment (opponents may counter and react) and even the rules are technically not fixed - take the Kobayashi Maru test situation as an example.

We argue that the development of highly innovative/uncertain and products is rather like an exploration journey. You have a vision where you want to end up and a 
general idea where your project is heading. However, neither can you know all the "moves" required to get there, nor can you accurately anticipate the effects and responses that one move will have in the future. Your expertise is your toolbox and it greatly helps in "playing your way through the project". Nevertheless the project is dependent on many unforeseeable events. In fact unknown unknowns (variables that are part of your problem/solution that you are neither aware off nor do you know their value) arise, serendipitous events present themselves, turning a complicated problem into a complex one - too complex to be planned out beforehand. We subsequently argue that sequential process models are not fitting for any innovative projects. [1,2].

The reference case [3] of designing a tangible game interface for racing games is used to extract reoccurring patterns during the design process and propose a method based on the experiences [4,5]

Our proposed method is based on abductive learning $[6,7,8]$ and includes all involved disciplines from day one. This wayfaring model based on Steinert \& Leifer [9] aims to allow the rapid requirement dynamics that become necessary during the development process.

\section{Use of Wayfaring in the Example Case}

Our example case is based on the vision of developing a physical car model as a tangible interface for manipulating/shape a digital car model in a virtual car racing game. A description of the project and the technical solution can be found in [3] This vision as an overarching goal was given to the developers instead of a precise list of requirements on how the technical solution is supposed to look like and the project architecture was allowed to emerge. This meant that the space of possible solutions is open, ambiguous and uncertain. In our example case, the problem became to identify car parts in the physical model that are attached to each other and to recognize the assembled structure. We explored the solution space by trying to come up with as many possible solutions to the problems as possible (divergent thinking). Possible ideas for solutions included measuring resistance, power dissipation of wireless communication devices or pulsed light communication for identifying connected pieces. For determining the structure we looked into a centralized structure with one central part that collects the data from all assembled parts and a decentralized structure that only required the detection and identification of neighboring parts. However, with no, or only little, experience it is unknown to us which of the suggested ideas was feasible to pursue. We call these unsolved uncertainties unknown unknowns because these open questions emerged during the development process and were in itself unknown to us before engaging the problem. We argued that resistors were the cheapest, simples and most reliable proposition and that just detecting neighboring car parts simplified the algorithm. Furthermore, having to use a specific center part restricted the liberty of freely using any car part separately in the virtual game. However, these are only arguments based on limited experience and in order to converge on the most promising proposal one has to build and test ideas to gain new knowledge. This repeating cycle of divergent and convergent thinking with designing, building and testing ideas is called probing. The probing cycles lead to abductive learning where the test result leads to design requirement changes and ideas for the next probing cycle. In our case, we realized that the measurement of the 
resistors fluctuates significantly. This lead to changes in the programming of the microcontroller that processes the measurement. The abductive learning from repeating cycles of probing leads to a wayfaring of opportunistically finding one's way through the project. This means that the test results of the last probing cycle shape the future development. Figure 1a) shows the first test of the resistor principle. There we discovered that the idea is feasible and that three electrical connections between car parts are needed. This lead to the development of the setup shown in figure 1b) that uses BitSnap connectors that serendipitously already had exactly three electrical connections and allow the user to easily manipulate the physical car model. Testing these BitSnap connectors revealed that these are mechanically not sufficiently robust and not genderless, thus limiting the combinations of mountable car parts. This learning resulted in the development of the customized connectors shown in figure 1 c) and d) where the first version in figure 1c) turned out to be also not genderless and subsequently lead to the development of the second version in figure 1d). This train of subsequent probing cycles showcases the wayfaring journey that can be successive or dead ended.

Progress is achieved by the emergence of new ideas as a result of previous probing cycles. Therefore, it is important to minimize the time spent and to maximize the learning outcome for each probing cycle. This accomplished by concentrating on just testing the critical functions by building a low resolution prototype that is reduced to the properties that are necessary to only test the critical function. An example for this is the testing of the resistor principle as it is shown in figure 1a). The critical function was to find out how resistors can be used to identify connected parts unambiguously. To save time we compromised robustness, automation of the measurement, looks and compactness of the system to focus only on the critical function and thus used prototyping boards, header wires and ohmmeters that were readily available in the lab.

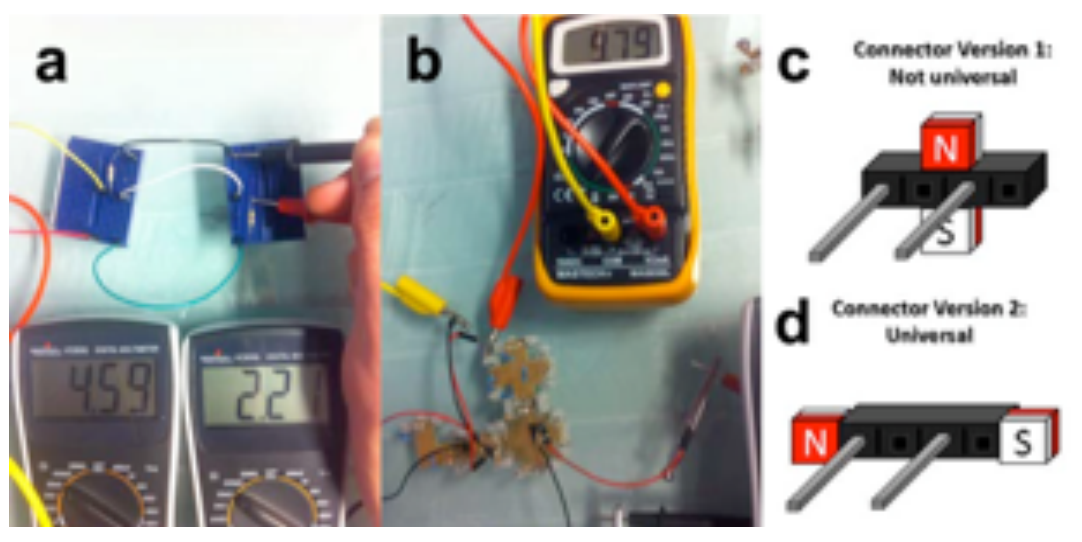

Fig. 1. a) first test of the resistor concept, b) testing with the BitSnap connectors, c) failed version of universal connector, d) successive version of a universal connector.

Imposing this train of thought to the entire project yields that prototypes that fulfill critical functions within different disciplines are merged as soon as they are available to test the system at large. The aim is to test and discover interdependencies. In our case, we combined the resistance measurement with a microcontroller, the 
information transmission to a $\mathrm{PC}$ and the virtual representation on the $\mathrm{PC}$ screen as soon as they were available in their most rudiment form. This means that all components from possibly different disciplines need to be prototyped simultaneously. Testing the entire system creates an interlaced knowledge between different disciplines. The structure recognition algorithm for example influenced the shape of the connectors and these changes had to be made in agreement with mechanical design of the car parts. This was possible because the developers of all disciplines were integrated from day one.

\section{A wayfaring approach to early stage concept creation}

In this part we describe a method that we derived from the project described above. The method has potential when finding and tackling previously unsolved engineering design problems that have no known existing solution. These problems are not necessarily complicated but rather complex according to Snowden and Boone [10]: they cannot be solved by asking experts to plan the final solution because they require the use of previously unproven and maybe even unknown concepts. In this context the development process becomes a wayfaring journey where the path towards fulfilling the vision emerges from making educated guesses and testing concepts, rather then a navigation journey along predefined waypoints. An optimum solution cannot be predicted when doing things that have never been done before. This method concerns only the early part of product development, the fuzzy front-end of concept creation, where the requirements of the product are not yet fixed. Figure 1 depicts such a wayfaring-inspired product development journey. This is a systematic and heuristic approach to developing something radically novel. The path to the end result will only be explored and discovered during the project. The journey consists of many probes. A probe is a circle of designing, building and testing of an idea or a prototype. In the figure 2, probes are depicted as multiple circles and may contain branching of ideas and prototypes on a multidisciplinary level or even dead ends. Each circle level corresponds to a role or a discipline in the project. At first, the team takes the bestguess direction based on the initial vision. Through multiple probing and prototype cycles the team then tries to find the big idea worth implementing. This journey can be long or short, but the main point is to learn fast with low-resolution prototypes. Through these prototypes one develops the requirements dynamically as perception of the problem and the vision of the solution will change during the journey. In a nutshell, we increase the degrees of freedom in the early design phase, develop requirements dynamically, and only then switch into classical engineering/project management mode.

While researching radical innovation projects, our chess analogy is lacking because in chess it is theoretically possible to calculate the move with the highest probability of winning the game. However, in the product design "game" the possible future moves, players, even the boundary conditions are often neither comparable nor foreseeable. There are unknown unknowns that create opportunities for extremely innovative solutions but also prevent us from predicting or simulating an optimum solution. In this analogy, the rules of the chess game can change without notice and we can only provide a journey overview in hindsight, roadmaps do not apply. The 
Hunter-Gatherer model by Steinert and Leifer [9] and Ingold [11] inspired this wayfaring concept.

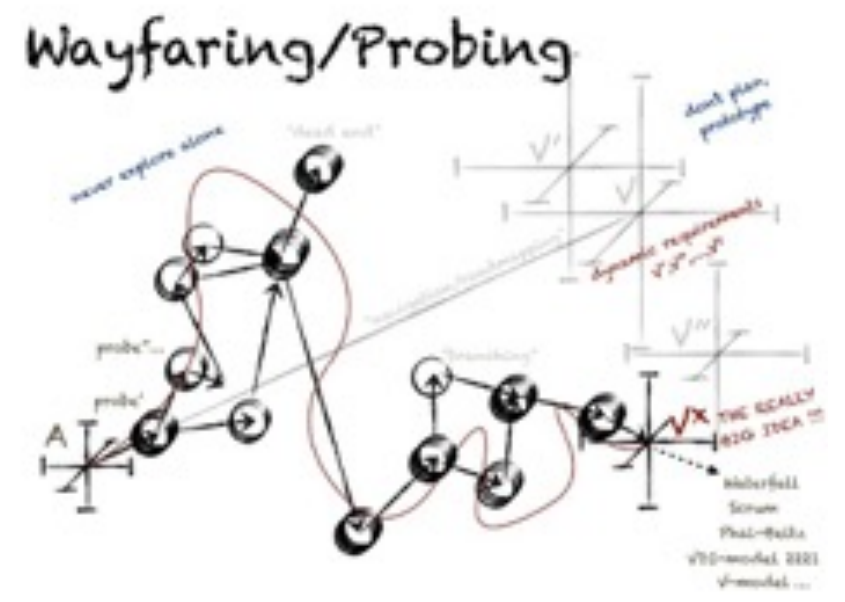

Fig. 1. Wayfaring journey in product development.

Many of our engineering problems are multidisciplinary and require interdependent knowledge between disciplines that cannot be covered by individuals or homogeneous teams. Two or more disciplines of the project are interdependent when design changes in one discipline lead to requirement adaptation in at least one other discipline. We argue that including team members or at least domain perspectives from all involved disciplines early in the project helps to reveal desirable and undesirable interdependencies already in early decision making phases. Even if actual deliverable input from every member is dispensable early on, the benefit of learning early overcomes the cost of participation. One of the greatest threats in new product development is the fear of failure [12]. According to Snowden [10] safe failing is identified as one of the cornerstones while innovating in the complex domain. The interlaced knowledge, developed through sense-making and justification of ideas to the other involved disciplines, is also beneficial when designing within one discipline while having the entire system in mind and thereby knowing when the other disciplines need to be taken into account and their input is needed [13]. This is a skill that can only be learned when combining all involved disciplines from the first day of the project.

The nature of trying out new concepts entails that outcomes cannot be guaranteed and some problems, opportunities and interdependencies are difficult, if not impossible, to foresee. When trying out something never attempted before we can no longer base our assumptions on past experiences and unexpected discoveries can arise. Snowden calls these discoveries unknown unknowns because we unknowingly discover something previously unknown [10]. In order to achieve these unexpected discoveries new experiences must be created from probing ideas. One of the ideas of probing is therefore to build and test prototypes that create completely new knowledge - knowledge that is impossible to accurately anticipate regardless of what our expectations may be. The concept of probing is depicted in Figure 2. Each probe is a prototype where new knowledge is deductively, inductively and/or abductively created and tested. The vision and requirements are then evolving dynamically until 
they are locked. The development cycle is executed through different roles of disciplines. Each probe is ideated through divergent thinking where open questions are asked in order to stimulate the creative process followed by convergent thinking, that evaluates and analytically benchmarks the ideas through proof-of-concept prototypes. The interesting interlaced knowledge lies in the boundaries of the different disciplines and presents the potential for serendipity discoveries.

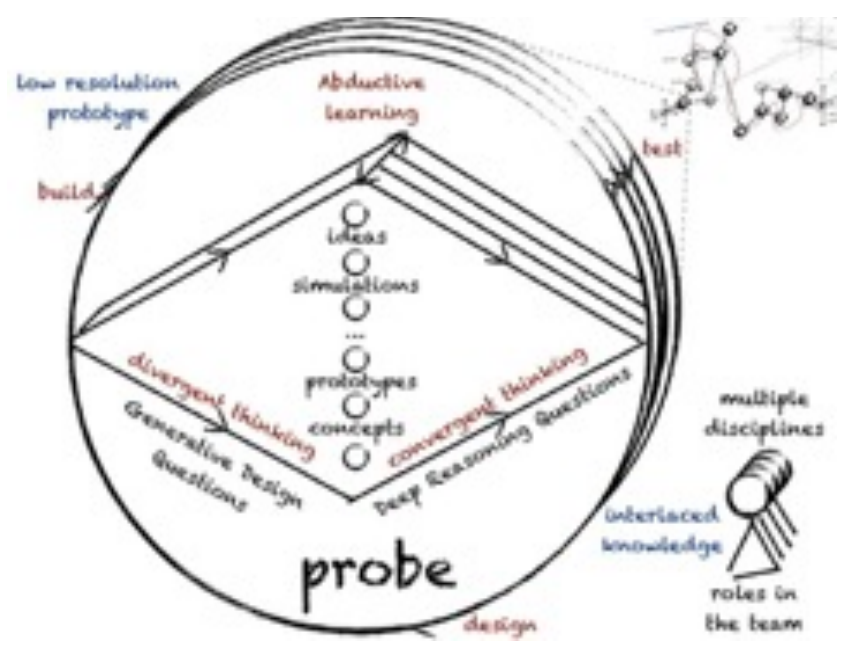

Fig. 2. Probing cycle

To continue with the chess analogy, we do not expect to win if we must plan all our moves (and anticipate the opponent's) in the beginning. However, if allowed to experiment and revert moves a thousand times during the game, it will quickly become a game of probing (or prototyping) multiple moves. Through not following an optimal game strategy, this will eventually lead to overall winning the game in case of a complex game scenario. Because the cost of probing is minimal, it allows us to explore opportunities that are not immediately perceived as profitable. It leads to moves that would normally not be taken, to discoveries that are normally not found, and may potentially lead to surprising and highly innovative ways of winning the game. Therefore, the aim must be to make the probing and the learning of ideas as low-risk (i.e. fast and cheap) as possible in order to create the experience needed to reflect, to understand the outcome, and then abductively reason and opportunistically choose the next step [14].

The notion is to put the focus on testing the most critical functions, thus leaving the development of the "nice to have" add-ons for later. It is preferable to utilize the resources for discovering the essentials and preferably fail there early. The probing removes uncertainty and an undiscovered problem is revealed before it forces undesired requirement changes at a later stage [15]. The testing usually involves building a low resolution prototype with the intention to either find the critical function or to build a prototype for user testing in order to avoid developing into an unnecessary direction. Low-resolution prototypes can be anything from cardboard models to Arduino hacks to proof-of-concept prototypes. Often developers have major problems in failing. Low-resolution prototypes in very fast iteration rounds do not resemble the finished object and are thus one way to allow and speed up 
experimentation. It seems to be inherent to human nature to fear failure, thinking it will cost too much. This can lead to a non-willingness to take risks and make cooperation hard with people from other disciplines. This skill of creative competence $[16,12]$ does not come naturally. This is why changing the mindset into one that favors building prototypes with the option of failing safely before planning is critical while developing new concepts. Hence, despite the natural fear of failing, the mindset should be biased towards building low-resolution prototypes in order to gain experience instead of thinking the idea through and remaining with doubt.

Another finding is to merge system components as soon as possible in order to tackle potential integration issues very early on. This follows the same line of thought as aiming to discover unknown interdependencies as early as possible. Whenever a component individually fulfills its critical function, it ought to be integrated with other components to test its critical function in the context of the whole system. So, even when the system can and is divided into modules, integration should be tested while changes to the system are still easily possible. We believe that there is no point in fully developing one component and then risking requirement changes in other components that would endanger the previous development. This requires quasisimultaneous prototyping to ensure that components can be merged. Thus in our context, simultaneous prototyping means understanding and probing ideas from multiple disciplines at the same time.

The main purpose of probing is to find solutions to the evolving problem by abductive reasoning and to continuously update the understanding of the problem. While probing different paths for the project one of the most important mindsets is to be opportunistic, to find, recognize and take chances that present themselves. Another benefit is the possibility to abandon disadvantageous concepts, "dead ends", in an early stage at the lowest cost and involvement possible. All in all, the wayfaring model calls for a bias towards action and learning in action.

\section{Conclusions of wayfaring}

We propose a method suitable for developing new products with a high degree of uncertainty. It is largely based on including all disciplines related to the product from the beginning on and iterative cycles of probing ideas by designing, building and testing prototypes. The intent of this approach is to discover unknown unknowns and unexpected interdependencies early in order to minimizing losses due to failure and to spot opportunities and hitherto unknown potentials. Both, the initial problem statement and the targeted project vision remain in flux much longer than usually. The relatively early requirement fixation stage becomes a delayed dynamic requirement evolution process. The decisions to fix the dynamic requirements are made based on gained and tested information, based on learning cycles trough low-resolution prototyping and probing. We believe the headway and learnings, both in terms of breadth and depths have been superior to pre-planned or more traditional process models. We thus invite the community to deploy and test this approach in the early, pre-requirement definition phase and to share their insights. 
Acknowledgments. We thank the project owner L. Nilsen, CEO of Metis Productions AS. This research is supported by the Research Council of Norway (RCN) through its user-driven research (BIA) funding scheme, project number 236739/O30.

\section{References}

1. Sanchez, R., \& Mahoney, J. T. (1996). Modularity, flexibility, and knowledge management in product and organization design. Strategic Management Journal, 17(S2), 63-76

2. Baldwin, C., \& Clark, K. (2000). Design Rules: The power of modularity. MIT Press

3. Reime, T. et al., Entertainment Computing--ICEC 2015: 14th International Conference, ICEC 2015, Trondheim, Norway, September 29 - October 2, 2015, Proceedings. Springer, 2015.

4. Eisenhardt, K.M. (1989). Building Theories from Case Study Research. The Academy of Management Review, 14(4), 532 - 550

5. Yin, R.K. (2013). Case Study Research: Designs and Methods. SAGE Publications.

6. Burks, A. W. (1946). Peirce's theory of abduction. Philosophy of Science, 13(4), 301-306.

7. Eris, O. (2004). Effective inquiry for innovative engineering design. Springer Netherlands.

8. Leifer, L. J., \& Steinert, M. (2011). Dancing with ambiguity: Causality behavior, design thinking, and triple-loop-learning. Information, Knowledge, Systems Management, 10(1), 151-173.

9. Steinert, M., \& Leifer, L. J. (2012). "Finding One"s Way': Re-Discovering a HunterGatherer Model based on Wayfaring. International Journal of Engineering Education, 28(2), 251.

10. Snowden, D. J., \& Boone, M. E. (2007). A Leader's Framework for Decision Making. Harvard Business Review, 69 - 76

11. Ingold, T. (2007). Lines: a brief history. Routledge.

12. Bandura, A. (1990). Perceived self-efficacy in the exercise of control over AIDS infection. Evaluation and program planning, 13(1), 9-17.

13. Türtscher, P. et al. (2008). Justification and Interlaced Knowledge at ATLAS, CERN. Organization Science, 25(6), 1579 - 1608

14. Schön, D. A. (1983). The reflective practitioner. Basic books New York.

15. Kriesi, C., Steinert, M., Meboldt, M., \& Balters, S. 2014. Physiological Data Acquisition for Deeper Insights into Prototyping. DS 81: Proceedings of NordDesign 2014, Espoo, Finland 27-29th August 2014.

16. Kelley, T., \& Kelley, D. (2013). Creative confidence: Unleashing the creative potential within us all. Crown Business. 